\title{
Characterization and Improvement of Neural Electrode-Tissue Interface
}

\author{
Xinyan Tracy Cui
}

Bioengineering Department, University of Pittsburgh, Pittsburgh, PA 15260

Neural prosthesis are devices used to restore, replace or bypass the lost neurological functions due to diseases or injuries[1]. One of the key components of such a system is a micro-fabricated neural electrode array, placed in the nervous system to directly interface with neurons[2,3]. Current arrays experience chronic failure due to the inflammatory tissue responses[4]. We examined the cellular and tissue responses around neural electrodes implanted in different regions of the nervous system including brain, spinal cord and dorsal root ganglia. Immunohistochemical staining of the tissue sections were used to identify the cell specific tissue responses. Confocal microscope imaging and image analysis were developed to quantitatively evaluate and compare the tissue parameters such as neuronal density, kill zone size, distribution of the glial cells. Neuronal damage and non-neuronal tissue build up around the implant are commonly found in all types of nervous tissue investigated. These phenomena may affect the stability and longevity of the neural recording and stimulation. Several biomimetic strategies are being investigated in our lab to improve the biocompatibility of neural electrodes and their long-term performance within the host brain tissue. The first strategy is immobilization of biomolecules (obtained from brain) onto the implant surface to promote attachment and growth of neurons while suppressing the adsorption of plasma protein and glia. Neural adhesion molecule L1 was covalent coupled to the surface of neural probes together with polyethyleneglycol(PEG)[5]. This coating has been characterized in vivo and showed improved neuronal health and reduced reactive glial response around the implant (Figure 1). The second strategy aims at covering the neural probe surface with a layer of neural stem/progentior cells(NPCs). We hypothesize that NPCs may serve to reduce the foreign body reaction by presenting a tissue friendly surface, and to repair implant induced injury and inflammation by releasing neurotrophic factors. Biomolecule laminin were coated on the surface before the cell seeding. The NPCs were able to adhere well on laminin immobilized probe surfaces and differentiate into neurons, astrocytes and oligodendrocytes. Finally the NPC seeded neural probes were implanted in the brain. The survival of the NPCs and the host tissue responses were investigated. Results show that the adhesion of NPCs to the neural probes was strong enough for the implantation (Figure 2). In addition, NPCs were observed to improve the astrocytic reaction around the implant site[6]. To better understand the electrode-tissue interaction, real time direct imaging of the electrode-tissue interface is desired. The ultimate solution to the chronic neural interface problems may be a combinatorial approach promoting the functional integration between the electrode and host tissue.

References:

1. Schwartz, A.B., Cortical neural prosthetics. Ann Rev of Neurosci, 2004. 27: p. 487.

2. Anderson, D.J., et al., Batch-fabricated thin-film electrodes for stimulation of the central auditory system. IEEE T Bio-med Eng, 1989. 36: p. 693.

3. Drake, K.L., et al., Performance of Planar Multisite Microprobes in Recording Extracellular SingleUnit Intracortical Activity. IEEE T Bio-med Eng, 1988. 35(9): p. 719.

4. Polikov, V.S., P.A. Tresco, and W.M. Reichert, Response of brain tissue to chronically implanted neural electrodes. J Neurosci Methods, 2005. 148(1): p. 1. 
5. Azemi, E., et al., Surface immobilization of neural adhesion molecule L1 for improving the biocompatibility of chronic neural probes: In vitro characterization. Acta Biomaterialia, 2008. 4(5): p. 1208.

6. Azemi, E., G.T. Gobbel, and X.T. Cui, Seeding neural progenitor cells on silicon-based neural probes. J Neurosurg. DOI: 10.3171/2010.1.JNS09313

7. This work is supported by National Institute of Health R01NS062019 and the Department of Defense TATRC grant WB1XWH-07-1-0716.

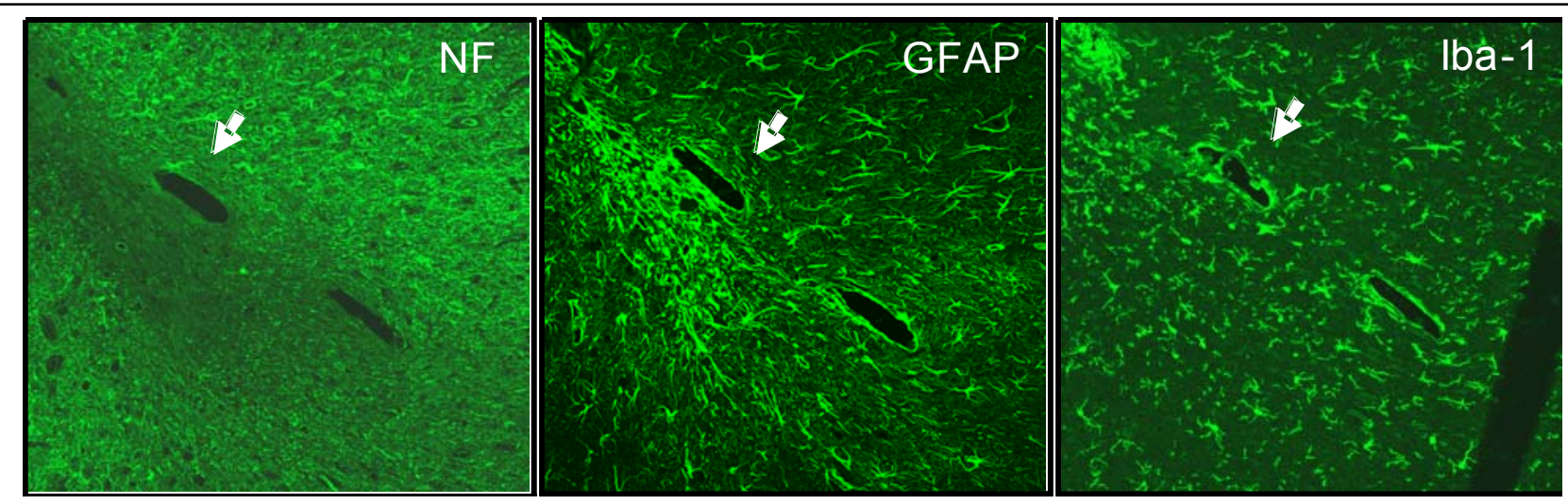

Figure 1. Host tissue response of a 4-shank NeuroNexus probe. The top side of the probe was coated with L1/PEG. The probe was implanted in the rat cortex for 1 week and removed after tissue fixation leaving 4 holes (only two shown under this magnification) in the tissue. The tissue sections were stained with anti-neurofilament (NF) to label neurons, anti-GFAP to identify astrocytes, anti-Iba-1 to identify microglia / macrophages. Arrows indicate the coated side. Distance between centers of the two holes $=200 \mu \mathrm{m}$.

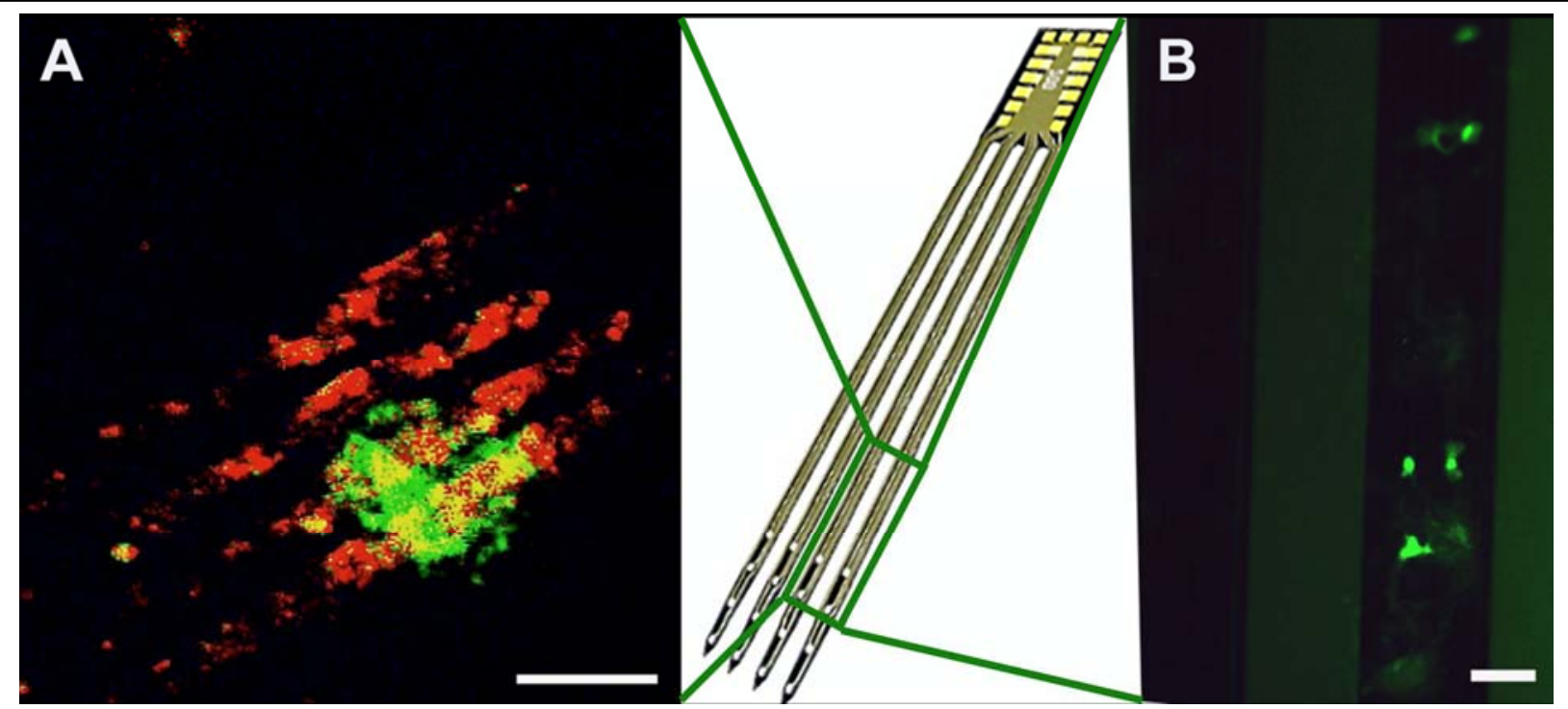

Figure 2. Examination of NPCs seeded on neural probes. A) 2-photon image of GFPNPCs (green) cultured in GM for 14 days on a laminin modified neural probe inserted in fixed brain tissue. 4-shank silicon based Michigan probes were used, and the probe auto-fluorescence is observed in red. B) Fluorescent image of a probe after 1-day post implant removal. Prior to implantation the GFP-NSC (green) were cultured for 14 days in GM and seeded on laminin immobilized probes. Images $\mathrm{A}$ and $\mathrm{B}$ are a zoom in of the selected box on the probe schematic in the middle (box and lines shown in green). Scale bars $=50 \mu \mathrm{m}$. 